INPLASY

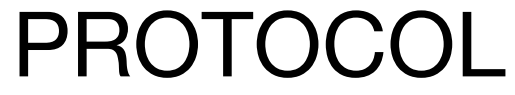

To cite: Sun et al. Effects of Physical Exercise on Attention Deficit Hyperactivity Disorder in Children: A Meta-analysis. Inplasy protocol 202140113. doi:

10.37766/inplasy2021.4.0113

Received: 21 April 2021

Published: 22 April 2021

Corresponding author:

Wenxin Sun

1305380127@qq.com

Author Affiliation:

Shanghai University of Sport

Support: 11DZ2261100.

Review Stage at time of this submission: Data analysis.

Conflicts of interest:

None declared.

\section{Effects of Physical Exercise on Attention Deficit Hyperactivity Disorder in Children: A Meta-analysis}

Sun, WX'1 Yu, MX²; Wang, $X^{3}$.

Review question / Objective: To explore the effects of physical exercise intervention on the cardinal symptoms of motor skills and executive function among children with attention deficit hyperactivity disorder (ADHD).

Condition being studied: Attention Deficit Hyperactivity Disorder in Children. Literature searches for randomized controlled trials.

Eligibility criteria: (1) patients (children or adolescents diagnosed with ADHD); (2) randomized controlled trials (RCTs); (3) type of intervention (PE programs, or increased PE in addition to treatment in the control group); (4) primary outcomes of ADHD symptoms of hyperactivity and inattention, and secondary outcomes of depression, social problems, motor skills, and executive function.

INPLASY registration number: This protocol was registered with the International Platform of Registered Systematic Review and Meta-Analysis Protocols (INPLASY) on 22 April 2021 and was last updated on 22 April 2021 (registration number INPLASY202140113).

\section{INTRODUCTION}

Review question / Objective: To explore the effects of physical exercise intervention on the cardinal symptoms of motor skills and executive function among children with attention deficit hyperactivity disorder (ADHD).

Condition being studied: Attention Deficit Hyperactivity Disorder in Children. 
Literature searches for randomized controlled trials.

\section{METHODS}

Participant or population: Children or adolescents diagnosed with ADHD (721). Intervention: Physical exercise.

Comparator: No treatment control.

Study designs to be included: Randomized controlled trials.

Eligibility criteria: (1) patients (children or adolescents diagnosed with ADHD); (2) randomized controlled trials (RCTs); (3) type of intervention (PE programs, or increased $P E$ in addition to treatment in the control group); (4) primary outcomes of ADHD symptoms of hyperactivity and inattention, and secondary outcomes of depression, social problems, motor skills, and executive function.

Information sources: PubMed, The Cochrane Library, Web of Science, Embase, CNKI, CBM, VIP and Wanfang databases.

Main outcome(s): Primary outcomes of ADHD symptoms of hyperactivity and inattention.

Quality assessment / Risk of bias analysis: The Cochrane bias risk assessment tools were used to evaluate methodological quality.

Strategy of data synthesis: The search strategy adopted a combination of subject words and free words and used the Boolean operations "AND" and/or "OR" to combine (topic or title) connections. It was subjected to repeated pre-checks and was supplemented by manual searches. The reference lists and related links of retrieved articles were examined to identify potentially eligible references for inclusion.

Subgroup analysis: Subgroup analysis was conducted for different indicators.

Sensitivity analysis: In the software, the literature was removed one by one.
Country(ies) involved: China.

Keywords: physical exercise, ADHD, children, meta-analysis.

Contributions of each author:

Author 1 - Wenxin Sun.

Author 2 - Mingxuan Yu.

Author 3 - Xing Wang. 